\title{
Various surgical techniques for the repair of injured vas deferens in rat experiments
}

\author{
Radek Stichhauer ${ }^{\mathrm{a}, \mathrm{b}}$, Jaroslav Koudelka ${ }^{\mathrm{a}, \mathrm{b}}$, Ales Ryskac, Helena Zivna ${ }^{\mathrm{d}}$, Milan Kaska ${ }^{\mathrm{b}}$
}

\begin{abstract}
Objective. The aim of this study was to evaluate whether the different vasovasostomy techniques can be performed using only the operating loupe in a rat model. The secondary aims were to evaluate the patency rate and inflammation of the vas deferens (VD) after contusion and the different vasovasostomy repair techniques.

Methods. A total of 40 male rats were divided into 4 groups based on the type of surgery: 1. contusion of the VD; 2. cutting of the VD and vasovasostomy with absorbable sutures; 3 . cutting and joining of the VD using absorbable sutures with an intraluminally situated lead fibre; and 4. cutting and joining of the VD using non-absorbable sutures with an intraluminally situated lead fibre. Ninety days after the surgery the VD was resected, patency and histopathological signs of inflammation in the VD were evaluated.

Results. All vasovasostomy techniques were successfully performed in all animals using only the operating loupe. The patency rate was $100 \%$ in the subgroup with contusion. Differences in the patency rates were found among the subgroups with vasovasostomy $(P=0.007)$. The patency rate was higher in the subgroup that underwent group 3 . Compared with vasovasostomies, contusion was associated with lower rates of inflammation $(P=0.02)$ and severe inflammation $(P=0.003)$. No differences were found among the subgroups of vasovasostomy techniques.

Conclusion. Contusion of the VD was not related to impairment in terms of patency. Vasovasostomy with an intraluminally situated lead fibre resulted in the highest patency rate among the standard vasovasostomy techniques.
\end{abstract}

Key words: vas deferens injury, inguinal herniotomy complication, operating loupe, vasovasostomy

Received: July 20, 2018; Accepted: October 24, 2018; Available online: November 15, 2018

https://doi.org/10.5507/bp.2018.069

(c) 2019 The Authors. This is an open access article licensed under the Creative Commons Attribution License

(https://creativecommons.org/licenses/by/4.0/).

${ }^{a}$ Department of Paediatric Surgery, University Hospital Hradec Kralove, Czech Republic

${ }^{b}$ Academic Department of Surgery, Faculty of Medicine in Hradec Kralove, Charles University, Hradec Kralove, Czech Republic

'Fingerland Department of Pathology, Faculty of Medicine in Hradec Kralove, Charles University and University Hospital Hradec Kralove, Czech Republic

${ }^{d}$ Radioisotope Laboratories and Vivarium, Faculty of Medicine in Hradec Kralove, Charles University, Hradec Kralove, Czech Republic Corresponding author: Radek Stichhauer, e-mail: radek.stichhauer@fnhk.cz

\section{INTRODUCTION}

Repair of inguinal hernia represents one of the most frequent general surgical operations in newborns, infants, and toddlers ${ }^{1}$. Different kinds of complications might occur after primary inguinal herniotomy, with a recurrence rate of $2.3 \%$ and a testicular atrophy rate of $2.7 \%$ in very small children ${ }^{2}$. Injury to the vas deferens (VD) represents one of the most common complication of inguinal hernia repair in newborns and toddlers ${ }^{2-4}$. Some kinds of VD injury could be detected in $0.8-2.0 \%$ of the cases ${ }^{5}$.

An unrecognized and untreated VD injury might be associated with serious long-term consequences such as infertility. This finding is supported by the fact that the prevalence of infertility in persons who underwent inguinal hernia repair is higher than that in the general population $^{6-8}$. In addition, the structures of the VD have unexpectedly been found to be parts of the resected hernia sac during histopathological evaluation in $0.1-1.7 \%$ of the cases ${ }^{9}$. Therefore, performing routine histopathological evaluation of the resected hernia sac in children was recommended; however, it has not reached clinical application owing to its low effectiveness ${ }^{9-12}$.

Although the different vasovasostomy techniques for VD injury repair in adults have been previously described in detail, there is a paucity of information about the appropriate surgical technique for neonates, infants, and toddlers ${ }^{13-17}$. An ideal technique for the paediatric population should be feasible without the use of microsurgery, as it is not commonly performed in standard paediatric surgery departments. Moreover, the ideal surgical technique should be associated with a high rate of VD patency and a low rate of complications, mainly inflammation.

To address these problems, the use of models of small laboratory animals seems to be ideal owing to the size similarity of their VD with that of neonates, infants, and toddlers. Some of studies seeking for the ideal techniques of vasovasostomy have been performed on rats, rabbits, and pigs and have suggested efficient and easy-to-perform surgical techniques for VD injury repair ${ }^{14,18-25}$. However, none of them has considered VD injury repair from a complex clinical point of view that encompasses the fea- 


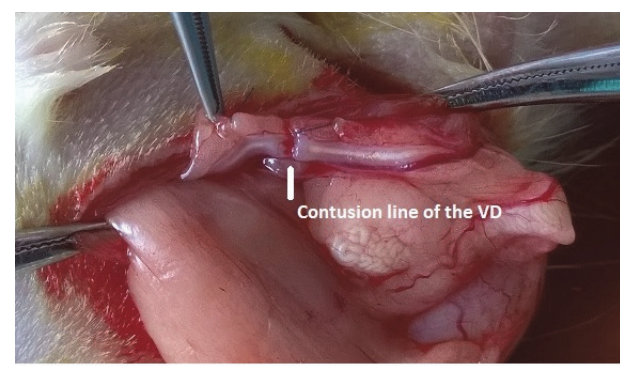

a

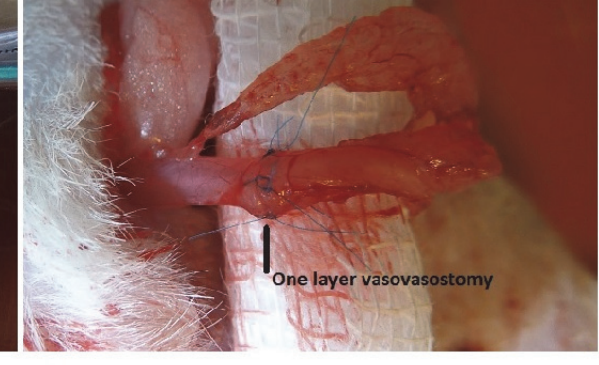

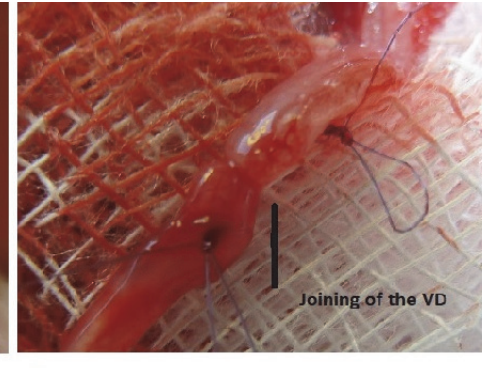

Fig. 1. Photographic schema (magnification: $\times 14$ ) of the contusion of the vas deferens.

(a), operation technique 1 (b), and operation techniques 2 and 3 (c).

sibility of surgery with the use of only the operating loupe, and the difference between contusion of the VD and the results of vasovasostomy techniques in terms of patency and inflammation rates.

Therefore, the primary aim of this study was to evaluate whether the different vasovasostomy techniques can be performed in a rat model using only the operating loupe. The secondary aim was to evaluate the patency rate and flow rate of the VD after contusion and the different vasovasostomy repair techniques in the rat model. The last aim was to evaluate which surgical procedure will be associated with the smallest inflammatory response.

\section{MATERIALS AND METHODS}

\section{Experimental Protocol}

The vivarium (CZ 527600 77) has and has been certified as a facility for the use of laboratory animals (see 3OZ21773 / 2011-17214, 144879/2011-MZE-17214 of 8 August 2011 for a period of 5 years and further st. 16OZ16371 / 2016-17214, No. 41058/2016-MZE-17214, of 27 July 2016 for a period of 5 years.

All animals received humane care in accordance with the guidelines set by the institutional Animal Use and Care Committee of Charles University, Faculty of Medicine in Hradec Kralove, Czech Republic. The protocol of the experiment was approved by the same committee (No. 31441/2015-3 and 1315-41795/12-30).

\section{Animals}

Experimental study was performed using 14-week-old male outbred Wistar rats (VELAZ s.r.o., Prague, Czech Republic) housed under standardized conditions (12-hour light/12-hour dark cycle, temperature $22 \pm 2{ }^{\circ} \mathrm{C}$, air humidity $30-70 \%$ ) in animal facilities of the Vivarium of the Medical Faculty of Charles University, Hradec Kralove, Czech Republic, with free access to food and drinking water.

\section{Operation procedures}

The rats were divided into 4 groups according to the method of VD injury and its repair. Before surgery the rats were anesthetized by intraperitoneal application of a mixture of ketamine ( $40 \mathrm{mg} / \mathrm{kg}$, Calypsol; Gedeon-Richer, Budapest, Hungary) and xylazine (5 mg/kg, Rometar $2 \%$ inj. ad usum veterinarium, $20 \mathrm{mg} / \mathrm{mL}$; Spofa, Praha, Czech
Flow patency rate of the vas deferens $p=0.004$

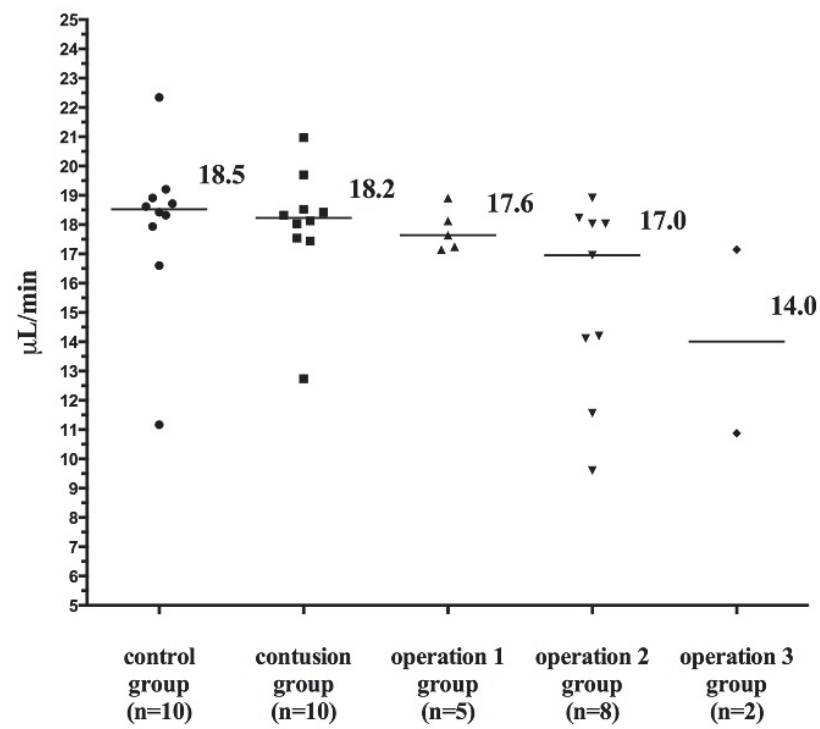

Fig. 2. Flow patency rate of the vas deferens.

Republic). The animals were operated under sterile conditions using basic minor surgical instruments with the aid of the operating loupe with 4-fold enlargement (NEITZ BLS 3, Tokyo, Japan).

After shaving the inguinal region, a $25-\mathrm{mm}$ subcutaneous incision was performed. The VD was isolated in full range while protecting the spermatic vessels.

In the $1^{\text {st }}$ group (Positive control), the VD was compressed for 2 seconds using Pean forceps, $20 \mathrm{~mm}$ distally from the epididymis junction (Fig. 1a). Thereafter, the VD was carefully returned to its correct position and the skin incision was closed. Then, the same incision and separation of the VD were done on the left side. The VD was carefully prepared without any injury, returned to its correct position, and the skin incision was closed. The left-sided VDs from this group were used as the control group (negative control).

In the $2^{\text {nd }}$ group (Technique 1 ), after isolation of the right VD $20 \mathrm{~mm}$ distally from the epididymis junction, the VD was cut off and the conventional one-layer vasovasostomy was performed (technique 1; Fig. 1b) using 3 single absorbable stitches (Vicryl R 8/0, Lot: AF5009; Ethicon, Somerville, NJ, USA). 
In the $3^{\text {rd }}$ group (Technique 2), after isolating the right VD $20 \mathrm{~mm}$ distally from the epididymis junction, the VD was cut off. In the 5-mm distance from the distal end of the VD, an insulin syringe $(0.33 \times 12 \mathrm{~mm}$, U 100 ; Chirana, Brno, Czech Republic) was inserted into the lumen of the VD by puncturing the VD. The lumen of the proximal end of the VD was threaded on the top of the same needle, and a $5-\mathrm{mm}$ length from the end of the VD was pierced out. The fibre of the absorbable suture (PDS 7/0, B Braun; Aesculap, Tuttlingen, Germany) was passed through the lumen of the needle and the needle was removed. The fibre retained inside the lumen of the VD was knotted extramurally to the proximal end of the VD. Then, the ends of the VD were joined, and the distal end of the fibre was knotted extramurally (technique 2; Fig. 1c). The VD was carefully returned to its correct position, and the skin incision was closed.

In the $4^{\text {th }}$ group (Technique 3 ), after isolating the right VD $20 \mathrm{~mm}$ distally from the epididymis junction, the VD was cut off. In the 5-mm distance from the distal end of the VD, an insulin syringe $(0.33 \times 12 \mathrm{~mm}$, U 100 , Chirana) was inserted into the lumen of the VD by puncturing the VD. The lumen of the proximal end of the VD was threaded on the top of the same needle and a $5-\mathrm{mm}$ length from the end of the VD was pierced out. The fibre of the non-absorbable suture (Prolene 7/0, Lot: KJQ015; Ethicon/Johnson \& Johnson, Somerville, NJ, USA) was passed through the lumen of the needle and the needle was removed. The fibre retained inside the lumen of the VD was knotted extramurally to the proximal end of the VD. Then, the ends of the VD were joined and the distal end of the fibre was knotted extramurally (technique 3; Fig. 1c).

The animals were housed postoperatively in the same conditions as preoperatively. An analgesic, acetylsalicylic acid $100 \mathrm{mg} / \mathrm{kg} \mathrm{BW}$, was administered after surgery. Twenty-one days after the first surgery, the fibres of nonabsorbable suture (technique 3 group) were removed during the second operation. The procedure was performed under the same general anaesthesia and using the same procedure as that in the first operation. Ninety days after the initial operation, all animals were sacrificed under the same general anaesthesia. Then the VD on the side of the intervention was resected in full length in all animals. In $1^{\text {st }}$ group - contusion of the VD, the VD was excluded on both sides. The VDs from the left side (dissection of the VD only) were used as the negative controls. Animals were sacrificed after the resection of biological material using a high dose of anaesthetic.

\section{Flow rate measurement}

The injured and control VDs were resected in full length. Then, a $20 \mathrm{~mm}$ length of the VD starting at 15 $\mathrm{mm}$ from the epididymis junction was resected. At the centre of this resected part of the VD, a line of previous artificial injury was detected. The lumen of each of these VD parts was threaded into an insulin syringe $(0.33 \times 12$ $\mathrm{mm}, \mathrm{U} 100$, Chirana) at a depth of $5 \mathrm{~mm}$ and fixed using micro-clips. Methylene blue solution was used to examine the flow rate of the VD. This solution was applied using the insulin syringe with a linear perfusion pump, at a basic constant flow rate of $2 \mathrm{~mL} / \mathrm{min}$ with a perfusion time interval of 10min (Microdialysis Pump - CMA/102, Solna, Sweden; flow rate $0.1 \mu \mathrm{L}-20 \mathrm{~mL} / \mathrm{min}$ ). The solution that passed through the part of the VD was collected and then weighed. The flow rates were calculated from the weight of the collected solution, time interval, and known density (denseness) of methylene blue solution.

\section{Histopathological examination}

All specimens were fixed in formalin, processed according to routine procedures, and embedded in paraffin. Three-micrometer histological sections were cut and stained with hematoxylin and eosin.

Morphologic evaluation in terms of the presence of inflammatory and severe inflammatory changes in the resected VD sections was performed by the same pathologist without previous knowledge about the particular type of injury. Findings were scored using a scale which evaluated the following criteria: dilatation of the VD lumen, scaring, stasis of the seminal fluid, inflammation, presence of foreign bodies, granulomas, and formation of a seminal fluid pseudocyst ("seminal fluid sac"). All these features were assigned a score as follows: 0 - absent, 1 mild, 2 - moderate and 3 - severe. A total sum score was calculated classifying changes to the VD (in points) as none to minimal ( 0$)$, mild (1), moderate to constricted (2), or as severe, resulting in total obliteration or damage of the VD (3).

\section{Statistical analysis}

The clinical characteristics are presented as medians [interquartile range (IQR)] for continuous variables. Categorical variables were compared using Fisher's exact test or chi-square test, as appropriate, and are presented as numbers (\%). The normality of data was tested using the D'Agostino and Pearson omnibus normality test. Because the flow rates were not normally distributed, the non-parametric Jonckheere-Terpstra test was used for the analyses. Differences were considered statistically significant at $P<0.05$. All $P$-values were from 2 -sided tests, and all statistical analyses were performed using SPSS 19.0 for Mac OS X (SPSS Inc., Chicago, IL, USA) and with GraphPad Prism 6.0h for Mac OS X (GraphPad Software, La Jolla, CA, USA).

\section{RESULTS}

\section{Clinical characteristics}

In total, 40 male Wistar rats were used in the experiment. The rats were divided into 4 subgroups on the basis of the type of surgery. The clinical characteristics are shown in Table 1. Differences in rat body weight in the first operation were not significant, the differences in the body weight gain between operations in subgroups was statistically significant $(P=0.03)$. Those did not affect the success of the individual techniques of the vas deferens reparation. The cut off and subsequent vasovasostomy of the vas deferens required a longer operating time than 
Table 1. Clinical characteristic of the rat subgroups.

\begin{tabular}{|c|c|c|c|c|c|}
\hline Characteristic & $\begin{array}{l}\text { Contusion } \\
\text { group } \\
(\mathrm{n}=10)\end{array}$ & $\begin{array}{l}\text { Operation } \\
\text { technique } \\
1^{\text {st }} \text { group } \\
(\mathrm{n}=10)\end{array}$ & $\begin{array}{l}\text { Operation } \\
\text { technique } \\
2^{\text {nd }} \text { group } \\
(\mathrm{n}=10)\end{array}$ & $\begin{array}{l}\text { Operation } \\
\text { technique } 3^{\text {rd }} \\
\text { group } \\
(\mathrm{n}=10)\end{array}$ & $P$ \\
\hline Body weight $^{1}[\mathrm{~g}$, median $(\mathrm{IQR})]$ & $414(395-426)$ & $455(428-499)$ & $426(403-444)$ & $423(398-451)$ & 0.06 \\
\hline Body weight $^{2}[\mathrm{~g}$, median (IQR)] & $481(461-530)$ & $557(511-601)$ & $534(506-549)$ & $511(482-544)$ & 0.03 \\
\hline Operation time [min, median (IQR)] & $13(12-23)$ & $24(22-28)$ & $20(18-23)$ & $21(20-24)$ & $\mathbf{0 . 0 2}$ \\
\hline
\end{tabular}

Abbreviations: IQR, inter-quartile range; Body weight ${ }^{1}$, body weight at the beginning of the experiment; Body weight ${ }^{2}$, body weight at the end of the experiment.

Continuous variables are compared using the non-parametric Kruskal-Wallis test. Statistically significant results are marked in bold. Continuous variables are presented as median (inter-quartile range).

simple contusion $(P=0.02)$. All 3 kinds of vasovasostomy techniques were performed in all operated animals using only the operating loupe.

\section{Patency of the VD}

The patency rate of the VDs was $100 \%(10 / 10)$ in the subgroup with contusion (positive control). The rate patency in this subgroup was higher than that in the subgroups with vasovasostomy [100\% (10/10) vs. $53 \%$ $(16 / 30), P=0.007]$. Differences in the patency rates were found among the subgroups with vasovasostomy [technique $1: 50 \%(5 / 10)$, technique 2: $90 \%(9 / 10)$, technique 3: $20 \%(2 / 10) ; P=0.007)]$. The patency rate was higher in the subgroup with technique 2 than in the subgroups with technique $1(P=0.05)$ and technique $3(P=0.002)$. No difference in the patency rate was identified between the subgroups with techniques 1 and $3(P=0.16)$.

A difference in the flow rate through the VDs was seen among the positive control subgroup, the subgroup with contusion, compared the subgroups with vasovasostomy (control: median $18.5 \mu \mathrm{L} / \mathrm{min}, \mathrm{IQR}$ 17.6-19.00; contusion: median $18.2 \mu \mathrm{L} / \mathrm{min}$, IQR 17.5-18.8; technique 1 : median $17.6 \mu \mathrm{L} / \mathrm{min}$, IQR $17.2-18.5$, technique 2: median $17.0 \mu \mathrm{L} / \mathrm{min}$, IQR $12.8-18.1$, technique 3: median 14.0 $\mu \mathrm{L} / \mathrm{min}$, IQR 10.9-17.2; $P=0.004$; Fig 2).

\section{Histopathological presence of inflammation in VD}

Histopathological evaluation of inflammation was evaluated in all resected VDs. The minimal inflammations were in the groups with contusion (positive control) and vasovasostomy technique 1 and for 90\% (9/10) and $70 \%(7 / 10)$ of VDs in the subgroups with vasovasostomy techniques 2 and 3, respectively. Contusion was associated with lower rates of inflammation or severe inflammation than vasovasostomies [inflammation: $30 \%$ (3/10) vs. $77 \%$ (22/26), $P=0.02$; severe inflammation: $0 \%(0 / 10)$ vs. $54 \%(14 / 26 \%), P=0.003]$. No differences in the rates of inflammation and severe inflammation among the groups with vasovasostomy techniques were identified [the rates of inflammation-technique 1: $80 \%(8 / 10)$, technique 2 : $78 \%$ (7/9), technique $3: 71 \%(5 / 7), P=0.92$; severe inflammation-technique 1: 40\% (4/10), technique 2: 67\% (6/9), technique 3 : $57 \%(4 / 7), P=0.50]$.

\section{DISCUSSION}

Although iatrogenic damage to the VD is not a frequent event, it might be associated with serious complications and long-term consequences. Therefore, all types of iatrogenic damage to the VD should be repaired using an appropriate method.

The main findings of this study were as follows: i) all vasovasostomy techniques were performed using only the operating loupe; ii) contusion of the VD is not related to its impairment in terms of patency; iii) vasovasostomy with an intraluminally situated lead fibre (technique 2) resulted in the highest patency rate among the vasovasostomy techniques; iv) the presence of inflammation in the VD was less frequent after contusion than after vasovasostomy; and v) no differences in the presence of inflammation in the VD were found among the different vasovasostomy techniques.

Repair of an inguinal hernia is one of the most frequent and routine paediatric surgical operations; however, it can be complicated by an injury to the VD in newborns, infants, and toddlers, particularly in acute surgeries. Experimental surgical methods, useful for mimicking situations in human clinical practice, have some limitations, such as, first, on the morphological level (use of an experimental animal); second, from a general biological perspective (some differences exist between the animal and human immune systems); and third, owing to the technical challenges of applying surgical reconstructive methods for the repair of VD injury ${ }^{8}$. The impossibility of using the microscopic method in cases of VD injury in a standard paediatric surgical department is one of the main problems hindering excellent repair. A few studies investigating the above-mentioned problems have been published. However, most of them did not focus on the details investigated in the current study. Our trial concentrated on various issues in the repair of VD injury, similarly to the results of the first published report ${ }^{21}$, which focused on the possibility of surgical success in paediatric surgical departments equipped with standard instrumentation. The idea and the results of this previous study were the sources of inspiration for our present investigation; thus, our experiment evaluated some of the same methods and other possibilities for VD reconstruction after injury.

Some of other recently published studies applied microscopic methods for VD repair. These techniques 
are based on the application of very special instruments, devices, and apparatuses. Some studies about the use of fibrin glue for the suture of transected VD have also been published $^{25}$. The results of microsurgical methods are very satisfactory; however, these techniques are performed at higher costs and take a longer time to implement ${ }^{15,16}$. The same holds true for methods of robotic-assisted microsurgical VD reconstruction after injuries arising from various causes ${ }^{17}$. All of these methods require a highly trained surgeon and very expensive equipment. For this reason, they are not available in standard paediatric surgery departments worldwide. On the contrary, the operating loupe is a basic surgical equipment. Injury to the VD during an operation for inguinal hernia is an emergency situation requiring urgent surgery. We were searching for a method that would be generally applicable. The results of our experiment proved that vasovasostomy could be performed using only the operating loupe and the results can be high successful in the case of using the technique 2. This conclusion is consistent with recent by us discussed publication ${ }^{21}$.

The question of whether contusion caused by a surgeon's fingers or by a small instrument can cause some dramatic pathological changes in the VD wall and lumen has been addressed previously. In these studies, contusion of the VD with the fingers of the surgeon or contusion created using a soft instrument for a short time only leads to inflammatory changes; in contrast, pressing with a mosquito Pean leads to obliteration of the VD (ref. ${ }^{7,26}$ ). The final finding of our study was that brief contusions created by a surgical instrument are not very dangerous for VD patency and cause very mild histological changes. Only the influence of a longer duration of pressure can cause some damage to the VD structures. However, gentle handling of the structures in the inguinal canal during an operation for inguinal hernia should be the gold standard for operating procedures in paediatric surgery.

The functional quality of the injured and repaired VD was assessed by measuring the VD patency. Herein, we verified that technique 2 is a useful and simple method of vasovasostomy based on leading the suture intraluminally while knotting it extraluminally. This type of vasovasostomy resulted in the highest the patency rate among the standard vasovasostomy techniques. Moreover, this technique was faster and easier to perform for a standardeducated paediatric surgeon using the operating loupe. The use of non-absorbable suture proved to be unsatisfactory, although the use of non-absorbable material in other studies has led to good results ${ }^{22}$. Although the fibre was removed, the postoperative functional and histological changes in the VD were the worst. Certainly, the role of repetitive surgical performance may have also played a role.

Less inflammation was observed in the positive control group with contusion of the VD, which was certainly due to the lower invasiveness of the procedure. All other groups (technique 1, 2 and 3 ) had a significantly higher level of damage. The severity of inflammation was rather similar among the VDs repaired using different tech- niques. Therefore, although differences in the assigned scores for the evaluation of postoperative morphological changes can illustrate changes associated with the direct effect of operation/injury to any type of tissue, the measurement of flow rate through the affected VD section seems to be a more appropriate and objective method for assessing the final function of the VD.

\section{CONCLUSIONS}

The vasovasostomy by absorbable sewing fibre situated in VD lumen and knotted extramurally can be performed with very good functional result using only the operating loupe. Contusion of the VD is not related to its impairment in terms of patency. Vasovasostomy with an intraluminally situated lead fibre resulted in the highest patency rate among the evaluated vasovasostomy techniques. The presence of inflammation in the VD was less frequent after contusion than after vasovasostomy; however, no differences in the presence of inflammation in the VD were found among different vasovasostomy techniques.

\section{ABBREVIATIONS}

VD, vas deferens; IQR, inter-quartile range; BW, body weight.

Acknowledgment: The authors are grateful Dagmar Jezkova (laboratory technician) and Vaclav Krejci, Kristyna Petkov, and Tereza Cizova (medical students) for their help with the laboratory work. This study was supported by a grant from the Grant Agency and SVV Grant Agency, Charles University, Czech Republic (Grant no. 1603/2015). Additional support was received from the Faculty Hospital in Hradec Kralove (a long-term organization development plan). The grant provider had no role in the study design, data collection and analysis, decision to publish, or preparation of the manuscript.

Author contributions: As the principal investigator, RS had full access to all study data and takes responsibility for the integrity of the data and the accuracy of the data analysis in tight cooperation with MK; MK, RS, JK, HZ, DJ, VK, KP, TC: study concept, design and experiments; AR: histological examination; MK, RS: manuscript drafting, critical revision; All authors checked and approved the final manuscript.

Availability of data and materials: All data, that is included in the analysis and from which the conclusions are drawn are available in the Department of Paediatric Surgery, Teaching Hospital, Sokolská 581, 50005 Hradec Králové, Czech Republic.

Conflict of interest statement: The authors state that there are no conflict of interest regarding the publication of this article. 


\section{REFERENCES}

1. Tovar JA. Hernias - inguinal, umbilical, epigastric, femoral and hydrocele. In: Puri P, Höllwarth ME et al. Pediatric Surgery. Berlin Heidelberg: Springer; 2006. p. 139-152.

2. Nagraj S, Sinha S, Grant H, Lakhoo K, Hitchcock R, Jonson P. The incidence of complications following primary inguinal herniotomy in babies weighing 5 kg or less. Ped Surg Int 2006;22:500-2. doi: 10.1007/s00383-006-1695-7

3. Baird R, Gholoum S, Laberge JM, Puligandla P. Prematurity, not age at operation or incarceration, impacts complication rates of inguinal hernia repair. J Ped Surg 2011;46:908-11. doi: 10.1016/j.jpedsurg.2011.02.059

4. Santacruz MG, Navarro JM, Goenechea AE Ceballos AC, Zaplana HS Cobo BJ. Low prevalence of complications of delayed herniotomy in the extremely premature infant. Ac Ped 2004;93:94-8. doi: 10.1111/ j.1651-2227.2004.tb00681.x

5. Jenkins JT, O’Dwyer PJ. Inguinal hernias. BMJ 2008;336:269-72. doi: 10.1136/bmj.39450.428275

6. Parkhouse $\mathrm{H}$, Hendry WF. Vasal injuries during childhood and their efect on subsequent fertility. Br J Urol 1991;67:91-5.

7. Shandling B, Janik JS. The vulnerability of the vas deferens. J Ped Surg 1981;16:461-4.

8. Tekatli H, Schouten N, van Dalen T, Burgmans I, Smakman N. Mechanism, assessment, and incidence of male infertility after inguinal hernia surgery: a review of the preclinical and clinical literature. Am J Surg. 2012;204:503-9. doi: 10.1016/j.amsurg.2012.03.002

9. Steigman CK, Sotelo-Avila C, Weber TR. The incidence of spermatic cord in inguinal hernia sacs from male children. Am J Surg Patho 1999;23:880-5.

10. Partrick DA., Bensard DD, Karrer FM, Ruyle SZ. Is pathological evaluation of pediatric hernia sacs justified? J Ped Surg 1998;33:1090-4.

11. Miller GG, McDonald SE, Milbrandt K, Chibbar R. Routine pathological evaluation of tissue from inguinal hernias in children is unnecessary. Can J Surg 2003;46:117-9.

12. Kim B, Leonard MP, Bass J, Ruzhynsky V, de Nanassy J, Guerra L. Analysis of the clinical significance and cost associated with the routine pathological analysis of pediatric inguinal hernia sacs. J Urol 2011;186:1620-4. doi: 10.1016/j.juro.2011.03.071

13. Matsuda T, Muguruma K, Hiura Y, Okuno H, Schichiri $Y$, Yoshida $O$ Seminal tract obstruction caused by childhood inguinal herniorrhaphy: results of microsurgical reanastomosis. J Urol 1998;159(3):83740.
14. Meyer A, Blanc P, Balique JG, Kitamura M, Juan RT, Delacoste F. Laparoscopic totally extraperitoneal inguinal hernia repair: twentyseven serious complications after 4565 consecutive operations. Rev Col Bras Chir 2013;40:32-6.

15. Fischer MA, Grantmyre JE. Comparison of modified one- and twolayer microsurgical vasovasostomy. BJU Int 2000;85:1085-8.

16. Pasqualotto FF, Pasqualotto EB, Agarwal A, Thomas JAJ. Results of microsurgical anastomosis in men with seminal tract obstruction due to inguinal herniorrhaphy. Rev Hosp Clin Fac Med 2003;58:305-9.

17. Trost L, Parekattil S, Wang J, Hellstrom WJG. Intracorporeal robotassisted microsurgical vasovasostomy for the treatment of bilateral vasal obstruction occurring following bilateral inguinal hernia repairs with mesh placement. J Urol 2014;191: 1120-5. doi: 10.1016/j. juro.2013.11.107

18. Ho KLV, Witte MN, Bird ET, Hakim S. Fibrin glue assisted 3-suture vasovasostomy. J Urol 2005;174:1360-3. doi: 10.1097/01. ju.00000173941.87775.35

19. Jee SH, Hong YK. One-layer vasovasostomy: microsurgical versus loupe assisted. Fertil Steril 2010;94:2308-11. doi: 10.1016/j.fertnstert.2009.12.013

20. Chehval MJ, Martin SA, Alexander NJ, Winkelmann T. The effect of unilateral injury to the vas deferens on the contralateral testis in immature and adult rats. J Urol 1995;153:1313-5.

21. Ozen OI, Bagbanci B, Demitrola A, Karabulut R, Ozen O, Demirogullari B. A novel technique for vas deferens transection repairs. Ped Surg Int 2006;22:815-9. doi: 10.1007/s00383-006-1734-4

22. Dipaola G, Camoglio FS, Chironi C, Giacomello L, Cervellione RM, Cecchetto $M$. Reconstruction of the vas deferens: an experimental study on the rat. Minerva Urol Nefrol 2001;53:125-8.

23. Vrijhof EJ, De Bruine A, Zwindermann AH, Nijeholt AL, Kooles LH. The use of a newly designed nonabsorbable polymeric stent in reconstructing the vas deferens: a feasibility study in New Zealand white rabbits. BJU 2005;95:1081-5. doi: 10.1111/j.1464-410X.2005.0547.x

24. Junge K, Binnebosel M, Kauffmann C, Rosch R, Klink C, von Trotha K. Damage to the spermatic cord by the Lichtenstein and TAPP procedures in a pig model. Surg Endosc 2011;25:146-52. doi: 10.1007/ s00464-010-1148-1

25. Detweiler MB, Detweiler JG, Fenton J. Sutureless and reduced suture anastomosis of hollow vessels with fibrin glue: A review. J Invest Surg 1999;12:245-62.

26. Janik JS, Shandling B. The vulnerability of the vas deferens.II: The case against routine bilateral exploration. J Ped Surg 1982;17:585-8. 